\title{
A method to obtain the quantitative orientation of semicrystalline structures in polymers by atomic force microscopy
}

\author{
V. Speranza, S. Liparoti*, R. Pantani \\ Department of Industrial Engineering, University of Salerno - via Giovanni Paolo II, 132 - Fisciano (SA), Italy
}

Received 17 May 2021; accepted in revised form 16 July 2021

\begin{abstract}
Molecular orientation can determine the final properties in polymer parts during processing: in optoelectronic devices, the emission efficiency is strongly dependent on the orientation of the emitter materials; mechanical performances in polymer parts depend on the orientation and dimension of crystalline structures. A simpler and faster method to obtain the quantitative orientation of crystalline structures, based on atomic force microscopy, is introduced as a powerful alternative to the techniques mentioned above. This method is based on the acquisition of topographical maps along with the sample thickness and applying the directionality analysis to each map to obtain the distribution of orientation on the map. Such a distribution was analyzed following two approaches: the first one is based on Herman's analysis; it is quite similar to the one adopted for calculating the Herman's factor from the wide-angle X-ray scattering. The second one is simpler; it is based on the standard deviation of the distribution. Both approaches allowed the determination of an orientation parameter: the orientation parameter was close to 1 in the regions where a high number of oriented fibrils were found, vice versa, the orientation parameter was close to zero where spherulites were found. The orientation parameter was found highly consistent with Herman's factor for injection molded samples obtained with different mold temperatures, thus with different distributions of orientation and morphology.
\end{abstract}

Keywords: material testing, polypropylene, orientation, atomic force microscopy, directionality

\section{Introduction}

Molecular orientation, induced by injection flow, stretching operation, etc., influence different properties in polymeric products. Controlling the local anisotropic structures of various components is necessary to fabricate and design good performance polymer products [1].

The macroscopic optoelectronic properties of thin films made of polymeric organic semiconductors rely on the inherent anisotropy of their molecular structure. This has been observed in the performance of optoelectronic devices such as organic field-effect transistors (OFETs), organic light-emitting diodes (OLEDs), and photodetectors. In OLEDs, the emission efficiency is strongly dependent on the orientation of the emitter materials. Therefore, the development of techniques that enable the control of thin film deposition on the macroscopic scale, with molecular-scale control of orientation, is of the utmost importance for optimizing device performance [2].

The mechanical performances of polymeric parts also heavily depend on the orientation and dimension of the crystalline structures. Several studies showed an increase of mechanical properties on decreasing the fiber diameter [3]. Tan and Lim [4] confirmed a significantly increased Young's modulus for electrospun poly(l-lactic acid) (PLLA) fibers when diameters decreased. The same trend was observed 
for poly(2-acrylamido-2-methyl-1-propanesulfonic acid) and polyacrylonitrile electrospun fibers [5]. The intrinsic orientational order developed during electrospinning is expected to be a key factor determining electrospun fibers' properties and can be the primary reason for the fiber diameter-dependent thermal and mechanical properties. Therefore, birefringence can macroscopically reflect the degree of molecular orientation of amorphous polymer products. This technique is widely adopted to determine the residual stresses in the injection molding samples $[6,7]$. The orientation degree in the polymer chain can also be measured by infrared dichroism $[1,8$, 9]. X-rays are also widely adopted for orientation determination [10], especially the wide-angle X-ray scattering through Herman's factor [11]. All these techniques are recognized to be efficient in determining the macromolecular orientation. However, these techniques have to be coupled with other analytical techniques to have information about the correlation between the molecular orientation and the other properties, such as crystalline structure and morphology.

In this paper, atomic force microscopy (AFM) is explored as an additional route to describe the orientation distribution within semicrystalline injection molded samples. In particular, the amplitude error signal is adopted to obtain information about structure orientation through an analysis named directionality. The results show that AFM can provide two kinds of information, the morphology and the orientation of the crystalline structures to the direction of the flow experienced by the polymer during the process.

\section{Experimental}

\subsection{Materials}

The polymer adopted for the analysis of molecular orientation is the isotactic polypropylene (iPP, T30G, Basell, Ferrara, Italy), whose characterization is already reported in the literature $[12,13]$.

\subsection{Process}

The molded samples made of iPP were produced by the injection molding process, conducted by a Negri Bossi 70 ton machine (Negri Bossi S.p.A., Cologno Monzese, Milano, Italy), with $220^{\circ} \mathrm{C}$ injection temperature, $72 \mathrm{MPa}$ packing pressure, $8 \mathrm{~s}$ packing time, $2.9 \mathrm{~cm}^{3} \cdot \mathrm{s}^{-1}$ flow rate. The description of the cavity
Table 1. Test code, temperature adopted for the cavity surface and heating duration.

\begin{tabular}{|l|c|l|}
\hline Test code & $\begin{array}{c}\text { Temperature } \\
{[\mathbf{K}]}\end{array}$ & Heating duration \\
\hline CIM & 298 & whole cycle time \\
\hline F80 & 353 & filling \\
\hline P80 & 353 & filling and packing \\
\hline F150 & 423 & filling \\
\hline P150 & 423 & filling and packing \\
\hline
\end{tabular}

is also reported in previous work [11]. The temperature of the cavity surface was locally modulated by a heating device, whose description is reported elsewhere $[14,15]$. Such a heating device allows reaching the selected temperature ( 423 and $353 \mathrm{~K}$ ) on the cavity surface within 2 seconds and also permits to keep constant this temperature for the desired duration (as long as the filling stage or filling + packing).

Molded samples obtained in conventional conditions, namely CIM, with $298 \mathrm{~K}$ temperature of the whole mold, were also produced. Table 1 reports the test codes adopted in the following.

\subsection{Optical microscopy}

Leica slit microtome (mod. 625, Leica Biosystem, Buccinasco, Milan, Italy) was adopted to obtain thin slices, $100 \mu \mathrm{m}$ thick, that were observed by polarized optical microscope (Olympus BX51, Olympus Italia S.R.L., Segrate, Italy). The slices were oriented with an angle of $45^{\circ}$ with respect to the crossed polarizers.

\subsection{SAXS and WAXS}

Small and wide-angle scattering and diffraction apparatuss (SAXS and WAXS, Diamond Light Source, Harwell Science and Innovation Campus, Didcot, Oxfordshire, UK) was adopted to analyze the orientation of the samples produced by the injection molding process. In particular, Herman's orientation factors for (040) and (110) planes were evaluated from each acquired spectrum, adopting the Wilchinsky [16] equation. The $c$-axis orientation was evaluated adopting Equation (1), referred to the (110) $\alpha$ and (040) $\alpha$ reflections and the angle of $72.5^{\circ}$ between $b$-axis and the (110) $\alpha$ plane:

$$
\left\langle\cos ^{2} \sigma\right\rangle=1-0.901\left\langle\cos ^{2} \varphi_{040}\right\rangle-1.099\left\langle\cos ^{2} \varphi_{110}\right\rangle
$$


The reference direction was the flow direction. Equation (2) allows the calculation of the average orientation, for a set of $h k l$ planes, expressed as $\left\langle\cos ^{2} \varphi\right\rangle_{\mathrm{hkl}}$ :

$$
\left\langle\cos ^{2} \varphi\right\rangle_{\mathrm{hkl}}=\frac{\int_{0}^{\pi / 2} I(\varphi) \cos ^{2} \varphi \sin \varphi \mathrm{d} \varphi}{\int_{0}^{\pi / 2} I(\varphi) \sin \varphi \mathrm{d} \varphi}
$$

when $\varphi$ being the azimuthal angle, and $I(\varphi)$ the scattered intensity along the angle $\varphi$. Herman's orientation factor was calculated from Equation (3):

Herman's factor $=\frac{3\left\langle\cos ^{2} \sigma\right\rangle-1}{2}$

where Herman's factor is equal to -0.5 , orientation is perpendicular to the reference, i.e., flow, direction $\left(\varphi=90^{\circ}\right)$, if Herman's factor is equal to 1 , orientation is parallel to the reference directionn $\left(\varphi=0^{\circ}\right)$, and if Herman's factor is equal to zero, a random orientation is present.

\subsection{Atomic force microscopy}

The injection-molded samples were analyzed by atomic force microscopy (Multimode Dimension V coupled with Nanoscope V, Veeco, Santa Barbara, CA, USA, AFM in the following). OTESPA probe silicon cantilevers (Bruker, Billerica, MA, USA) with nominal radii of c.a. $7 \mathrm{~nm}, 300 \mathrm{kHz}$ resonance frequency, was adopted. The free amplitude was $50 \mathrm{mV}$. The probe-surface interaction forces lead to a reduction of the amplitude of the oscillation from its free value. The amplitude was adopted as a feedback parameter to map the topography (morphology) of the sample surface. During the acquisitions reported in this work, the setpoint amplitude was $60 \%$ of the free amplitude. The feedback mechanism kept the amplitude constant by adjusting the vertical position of the sample holder to compensate for the topography variations $[17,18]$. The difference between the setpoint amplitude and the target amplitude gives the amplitude error. All maps were acquired with a $0.5 \mathrm{~Hz}$ scan rate and 256 points per line. Bruker NanoScope software, version 7.30, was adopted for map acquisitions, and NanoScope Analysis software 1.8 was adopted to prepare the image for the analysis by Fiji, described below.

Each map's area was selected based on the mean dimension of the structures to analyze a significant amount of structure for each acquisition (at least 20 for each map). Areas of $70 \times 70 \mu \mathrm{m}$ were adopted in the spherulitic and transitional regions, characterized by structures with dimensions larger than $10 \mu \mathrm{m}$; areas of $10 \times 10 \mu \mathrm{m}$ were adopted in the shear layer, where nano-metric structures are present. The selection of the scanning area allowed analyzing homogeneous areas (composed of a single morphology), more important, where small structures were detected (i.e., fibrils), smaller areas allowed a better resolution (keeping constant 256 points per line). At each position, at least three maps were acquired.

The amplitude error maps were analyzed by Fast Fourier transform (FFT). For typically digitized AFM scans, the values of $x$ and $y$ are quantized, whereas $z$ is the signal to be analyzed. One can transform 2D AFM images from real space to reciprocal space, adopting FFT, as given in Equation (4) [19]:

$$
\begin{aligned}
& F\left(k_{\mathrm{x}}, k_{\mathrm{y}}\right)=\left(\frac{L}{N}\right)^{2} \sum_{\mathrm{x}=0}^{\mathrm{N}-1} \sum_{\mathrm{y}=0}^{\mathrm{N}-1} z(x, y) . \\
& \cdot\left(\cos \left(2 \pi\left(\frac{k_{\mathrm{x}} x}{N}+\frac{k_{\mathrm{y}} y}{N}\right)\right)+i \sin \left(2 \pi\left(\frac{k_{\mathrm{x}} x}{N}+\frac{k_{\mathrm{y}} y}{N}\right)\right)\right)
\end{aligned}
$$

where $F\left(k_{\mathrm{x}}, k_{\mathrm{y}}\right)$ is the FFT of $z(x, y), L$ is the scan size of the AFM image, and $N$ is the number of pixels, i.e., digitized data points per line. The FFT transformed version of the reciprocal space is difficult to be used cause the information is given in a $2 \mathrm{D}$ plane. It is possible to extract a linear pattern from the $2 \mathrm{D}$ transform by the Fiji plug-in Directionality (http://fiji.sc/Fiji, Ashburn, VA), which follows the procedure described by Liu [20]. The Directionality plug-in calculates the spatial frequencies within an image given a set of radial directions. The method generated normalized histograms revealing the number of structures oriented along a certain direction present between 0 and $180^{\circ}$ with a bin size of $1^{\circ}$. In the cases proposed in this work, $90^{\circ}$ corresponds to the flow direction during the injection molding process.

\section{Results and discussion}

The samples produced by injection molding coupled with the heating device for the local modulation of the temperature on the cavity surface are characterized by a peculiar morphology. Figure 1 shows the morphology developed within the injection-molded samples obtained in different conditions: the CIM condition corresponds to the traditional process during which the temperature of the whole mold has been kept at $298 \mathrm{~K}$, the second condition corresponds 


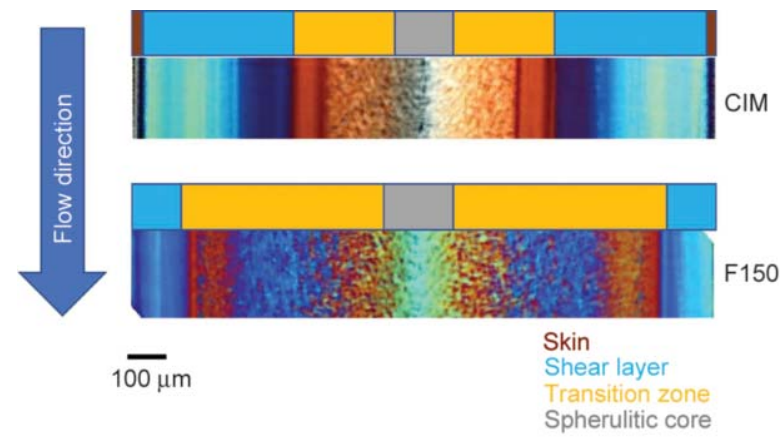

Figure 1. Micrographs obtained by polarized optical microscope of the CIM and F150 samples. Different colors are assigned to different morphologies.

to the activation of the heating device, which reaches $423 \mathrm{~K}$ temperature, during the filling stage (F150). The samples are characterized by different colored bands, each band corresponding to a certain degree of orientation [13]: the skin (brown), the shear layer (cyan), the transition zone (yellow), and the spherulitic core (grey). The shear layer is characterized by the highest degree of orientation, the spherulitic core by the lowest degree of orientation, the transition zone by an intermediate orientation value. The skin is present only when low temperatures (well below the crystallization one) are adopted for the cavity surface (the whole mold for the CIM case). In these conditions, the molecules suddenly quench when they touch the cavity surface, and a lower level of orientation and crystallinity degree is achieved.

The WAXS analyses allow obtaining quantitative information about the orientation distribution within

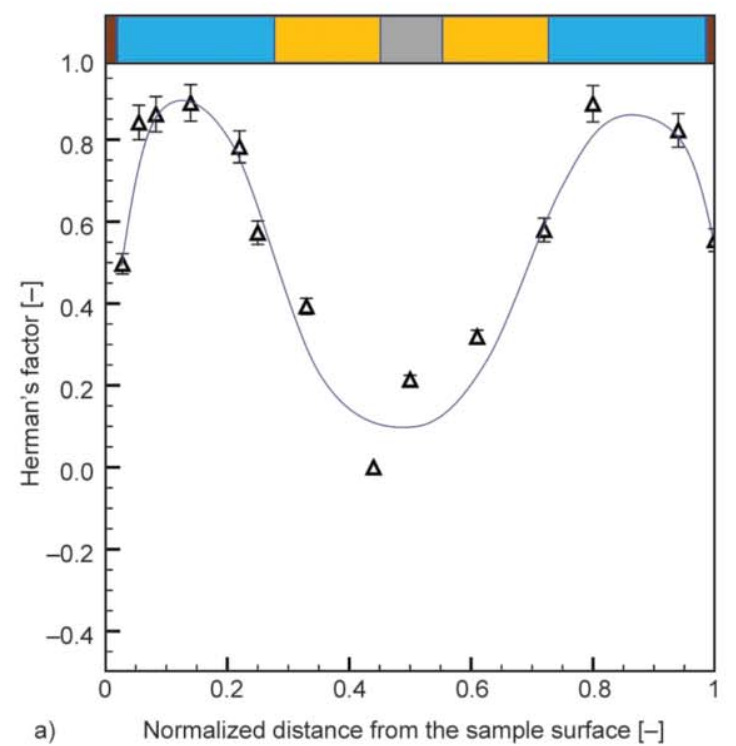

the samples (in the thickness direction). Figure 2 shows Herman's factor distribution along the sample thickness for the CIM and F150 samples. As mentioned above, in this work, the flow direction is the reference direction.

The distribution of Herman's factor confirms what was observed from the optical micrographs: the shear layers are characterized by the highest value of orientation for all the samples (Herman's factor ranges between 0.8 and 1 in the shear layers). The spherulitic core is poorly oriented: Herman's factor is close to 0 , which means that the spherulitic core is almost isotropic. In the transition zone, Herman's factor gradually decreases from the high value of the shear layer toward the low value of the spherulitic core (see Figure 2). The width of each layer strongly depends on the temperature field during the process. The CIM sample shows a wide shear layer, with larger values of Herman's factor in a wide region, almost $40 \%$ of the whole sample thickness. The CIM sample also shows a poorly oriented skin, with Herman's factor close to 0.3 at the sample surface. The sample F150 (Figure 2b) shows a narrower shear layer compared to the CIM sample, with Herman's factors of about 0.8 . The transitional zone is characterized by intermediate values of Herman's factor, in the range $0.3-0.5$, and covers most of the sample thickness, about $55 \%$.

The effect of the cavity surface temperature on the orientation can be explained on the basis of the flow and temperature fields that the polymer chains

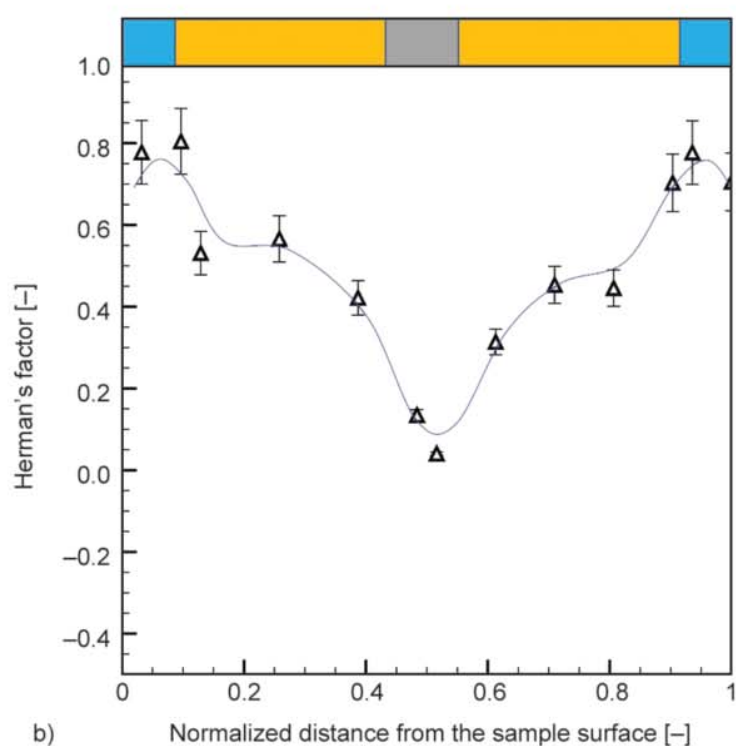

Figure 2. Distribution along the thickness direction of Herman's factor evaluated from WAXS analyses for the sample CIM (a); and F150 (b). 
experience during the solidification. When low temperatures are kept on the cavity surface, the polymer chains oriented by the strong flow fields quickly solidify and maintain their orientation along the flow direction. When high temperatures are kept at the cavity surface, the polymer chains have the time to relax their orientation before solidification. As a result, the residual orientation is lower than the sample obtained with low cavity surface temperatures [11].

The AFM analyses allow detecting the different morphological structures developed during the process. Figure 3 shows the morphology maps acquired by AFM in several positions along the thickness direction for the sample obtained with a cavity surface kept at $353 \mathrm{~K}$ during the filling (F80). FFTs of the amplitude maps are also shown in Figure 3. FFT provides a mathematical analysis of the image and produces a diffraction pattern, which provides a quantitative measure of the order present in the polymer template. SAXS and WAXS analyses are also shown for comparison.

AFM maps in Figure 3 show that the shear layer is composed of thin fibrils, with a mean thickness of about $250 \pm 10 \mathrm{~nm}$ (evaluated from the cross-section as the number of peaks detected along a given distance, see Figure 4a).

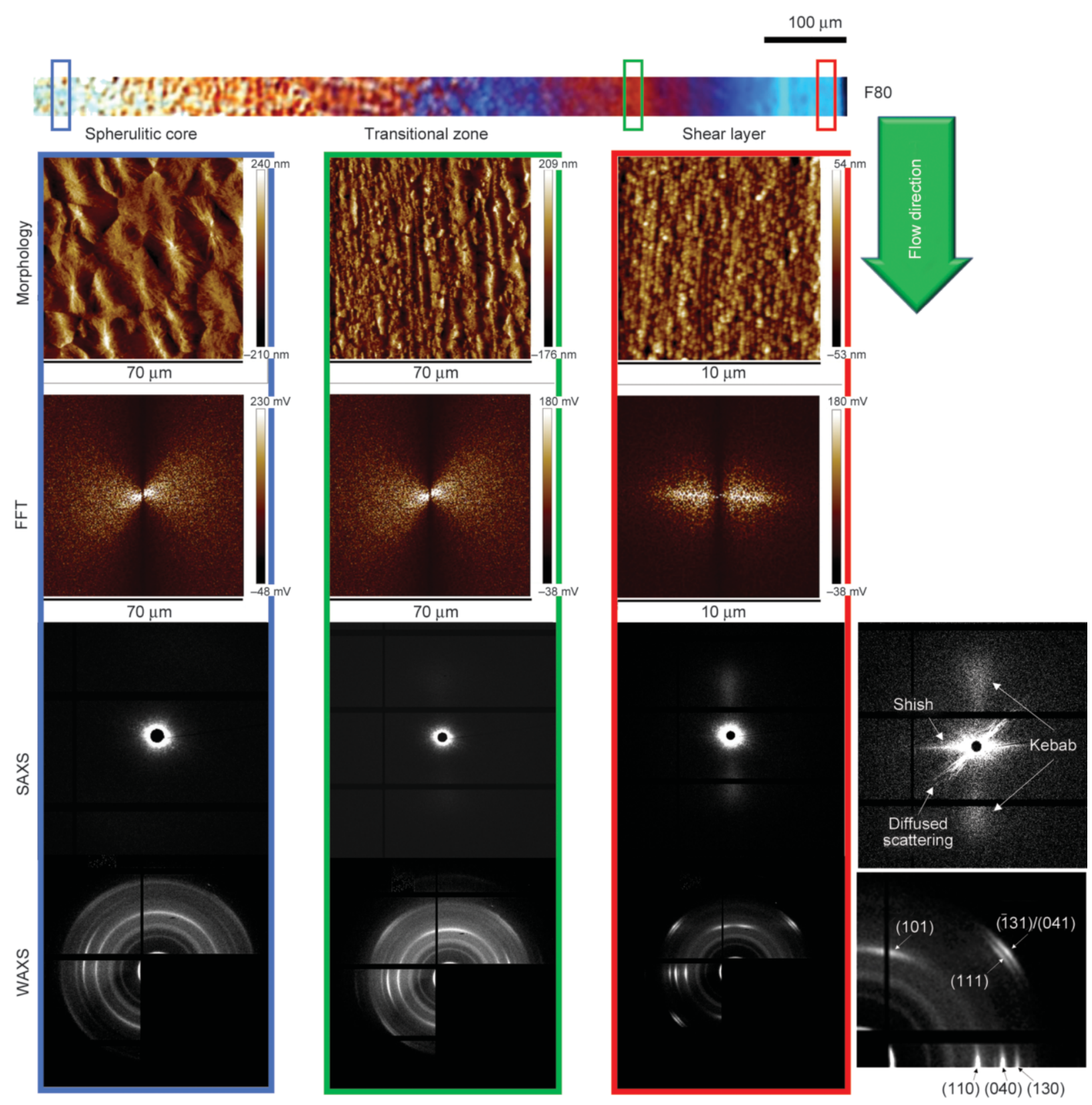

Figure 3. AFM maps, related to the morphology and the FFT of the amplitude error maps, of the sample F80. The optical micrograph of the sample is also reported for comparison (the sample core is on the left of the micrograph). SAXS and WAXS analyses are shown below each AFM map. 

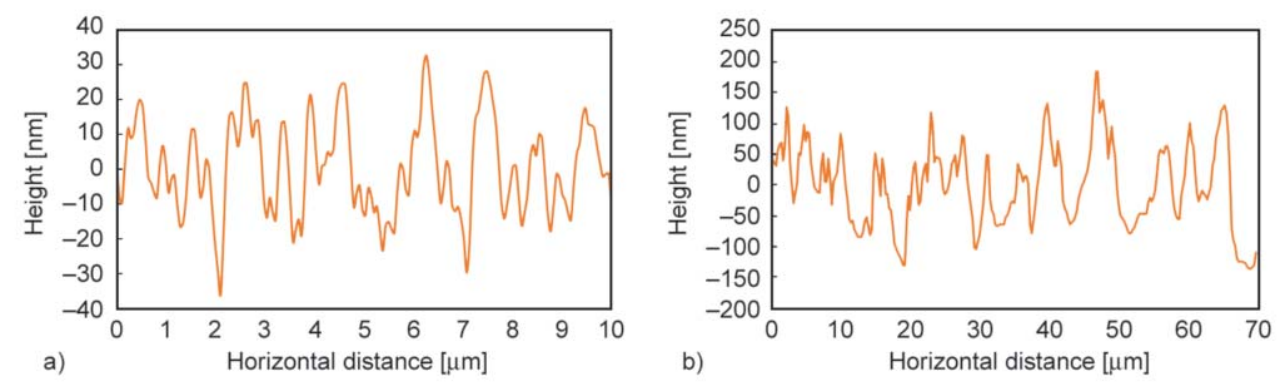

Figure 4. Cross-section height of the sample F80 in two positions: (a) the shear and (b) the transition layer.

Toward the sample core, the average thickness of the fibrils increases; furthermore, lateral structures, namely structures that grow along the orthogonal direction to the flow front, are detectable. This morphology is characteristic of the transition zone, where the average distance between adjacent fibrils is about $10 \pm 4 \mu \mathrm{m}$ (see Figure 4b). More isotropic structures are detectable in the sample core, namely spherulites, having a mean diameter of $18 \pm 2 \mu \mathrm{m}$. The mean diameter was evaluated as the diameter of an equivalent sphere having the same equatorial surface of the spherulite surface measured on the AFM map.

Figure 3 also shows the FFT of the amplitude error maps. The amplitude error map has been selected for this kind of analysis since it shows the highest contrast, being the error signal adopted for the instrument control. Using the linearity and rotation properties of FFT, a map can be decomposed into components lying in different directions. Extracting components in an angled band from the FFT map is equivalent to extracting the components lying in the corresponding orthogonal angle band from the original image. In the FFT map related to the shear layer, two scattering spots can be found on the equator, indicating the structure orientation along the flow direction due to the shear-induced crystallization during the injection molding process. In the FFT map of the sample core, the spread signal indicates the presence of more isotropic and unoriented structures; the FFT map of the transitional zone shows an intermediate behavior between the sample core and the shear layer. Figure 3 also shows the SAXS patterns in different positions along with the sample thickness. The patterns show an equatorial streak from the transition to the shear layer due to the formation of shish parallel to the flow direction. The meridional maxima are due to the formation of kebabs oriented perpendicularly to the flow direction [21]. Thus, the SAXS analyses confirm the orientation detected by FFT maps, even if the flow direction is orthogonal to the FFT analyses: the more oriented areas, namely the shear layers, are characterized by two scattering spots on the meridian, whereas the spread signal characterizes the sample core. The WAXS patterns, also shown in Figure 3, confirm this statement. Distinct arcs are detectable in all positions; they are ascribable to (110), (040), (130), (131)(041) and (111) reflections of the $\alpha$-form crystals. The orientation of the (110) reflection along the direction transversal to the flow, in the $\alpha$-form crystals, is due to the daughter lamellae, which grow approximately perpendicular to the mother lamellae oriented along the flow direction. In the sample core, four diffraction rings, due to $\alpha$-form crystals, are detectable in Figure 3, suggesting weak or no orientation. In this part of the sample, molecules relax the orientation and crystallize into more isotropic structures, the spherulites [22].

Figure 5 shows the WAXS 1D spectra along the azimuthal angle for the CIM and F80 samples. The spectra are referred to three positions along with the sample thickness: the shear layer, the transition zone, and the sample core, also shown in Figure 3.

For both samples shown in Figure 5, traces of the $\gamma$-crystals can be found in the shear layer and, in smaller amounts, in the transitional zone. The presence of $\gamma$-crystals is generally ascribed to an intense flow, characteristic of the shear and transitional layers. The $\alpha$-crystals are predominant in all the molding samples analyzed in this work.

Figure 6 shows the morphology maps, with the analysis of the cross-section height and several structures showing alignment along a certain direction, as evaluated by the Directionality plug-in of Fiji, for the CIM and F150 samples. The reconstruction of the signal obtained by Directionality analysis by a combination of Lorentzian and Gaussian functions $(G L(f))$, where $f$ is the direction, is also reported for each considered region along with the sample thickness.

In both cases, the shear layer is characterized by tightly packed oriented structures, having distances that 

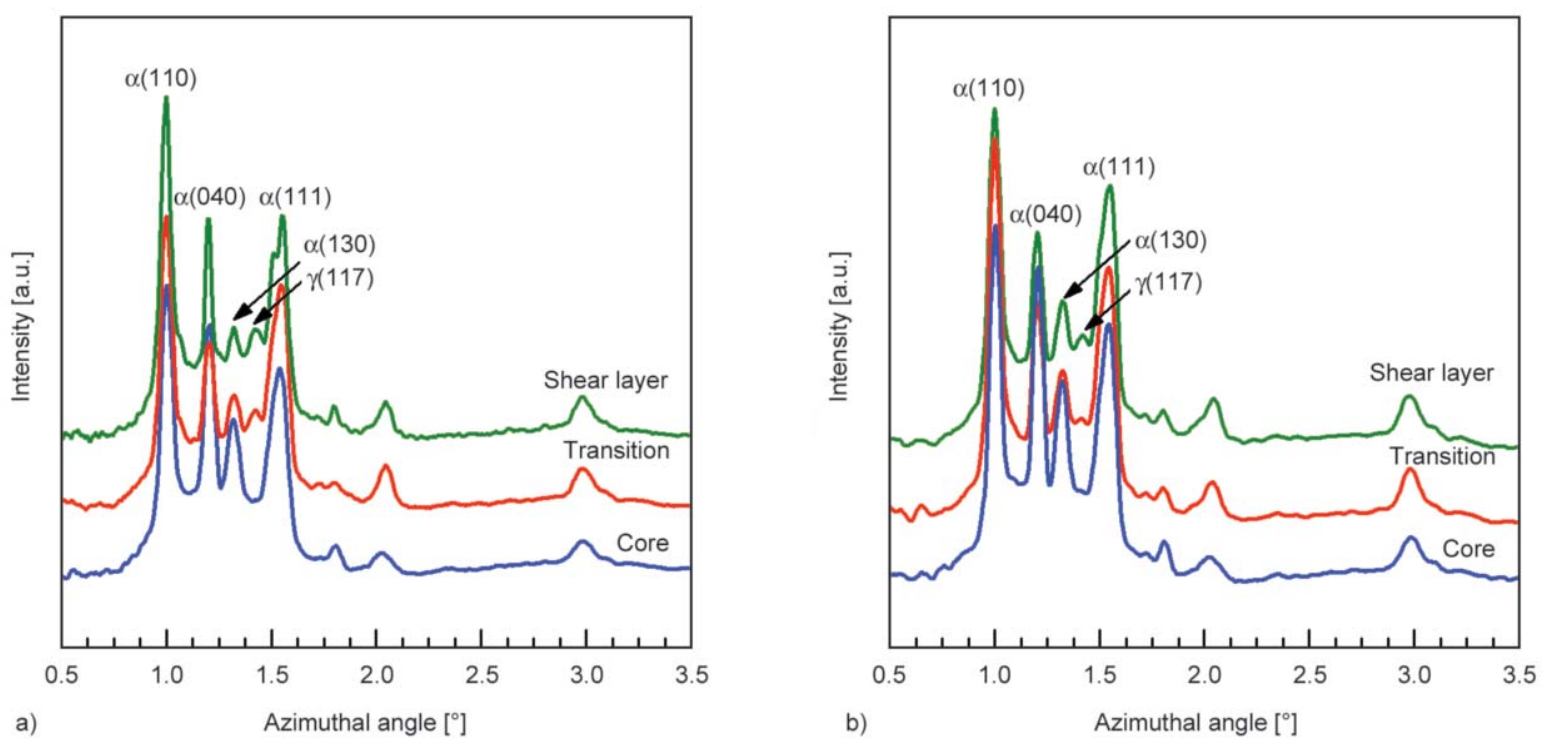

Figure 5. WAXS 1D spectra along the azimuthal angle for the (a) CIM, and (b) F80 samples. The spectra are referred to three positions along with the sample thickness: the shear layer, the transition zone, and the sample core, as indicated in Figure 3.

increase toward the sample core, from $200 \pm 50 \mathrm{~nm}$ in the shear layer to $2 \mu \mathrm{m}$ in the transitional zone. In the sample core, the distances between structures are in the order of $25 \mu \mathrm{m}$ and correspond to the spherulite diameters. Close to the sample surface, the CIM sample is characterized by randomly dispersed globes, which formation is due to a fast cooling, in the order of $100^{\circ} \mathrm{C} / \mathrm{s}$, occurring cause to the low temperatures of the mold; the fast cooling does not allow for the organization of molecules as spherulites or fibrils. Consistently, this last zone is characterized by low orientation, and the function $G L(f)$ appears broad. In the shear layer and the transition, the number of structures oriented along the flow direction (namely $90^{\circ}$ ) is high: the function $G L(f)$ is narrower for the shear layer and broader for the transition zone. Obviously, in the sample core, the function $G L(f)$ is broad.

The main purpose of this work is to obtain the orientation of each structure directly from AFM analyses. As mentioned above, the direction distribution is fitted by the function $G L(f)$. We applied a procedure similar to the one proposed by Herman for the analysis of WAXS to obtain a parameter, named $d$ in the following, which is similar to the $\left\langle\cos ^{2} \varphi\right\rangle_{\mathrm{hkl}}$, as given in Equation (5):

$$
d=\frac{\int_{0}^{\pi / 2} G L(f) \cos ^{2} f \sin f \mathrm{~d} f}{\int_{0}^{\pi / 2} G L(f) \sin f \mathrm{~d} f}
$$

Figure 7 shows the number of structures showing alignment along with a certain direction for F150 sample in two positions along the thickness. $G L(f)$ and the terms adopted in Equation (5) are also reported. At a normalized distance from the sample surface of 0.08 , which corresponds to the shear layer, the distribution is narrow, and the function $G L(f) \cdot \cos ^{2} f \cdot \sin f$ assumes almost the same values of the function $G L(f) \cdot \sin f$. At a larger normalized distance from the sample surface, i.e., at 0.23 , the distribution becomes wider and the function $G L(f) \cdot \cos ^{2} f \cdot \sin f$ becomes different from the function $G L(f) \cdot \sin f$.

Similarly to Herman's factor, the parameter $D$ can be expressed as given in Equation (6):

$D=\frac{3 d-1}{2}$

According to Equation (6), $D=0.96 \pm 0.04$ at a normalized distance from the sample wall of 0.08 , and $D=0.62 \pm 0.03$ at a normalized distance from the sample walls of 0.23 . Figure 8 shows the comparison between Herman's factor and the parameter $D$ at different positions along the thickness for the sample F150. When the parameter $D$ is close to 1, the structures are oriented along the flow direction. Unoriented structures are characterized by a parameter $D$ as close as possible to 0 . Figure 8 also shows that parameter $D$ is consistent with Herman's factor: $D$ is high, close to 1 , in the shear layer and gradually decreases down to 0.3 toward the sample core. Figure 9 shows the 

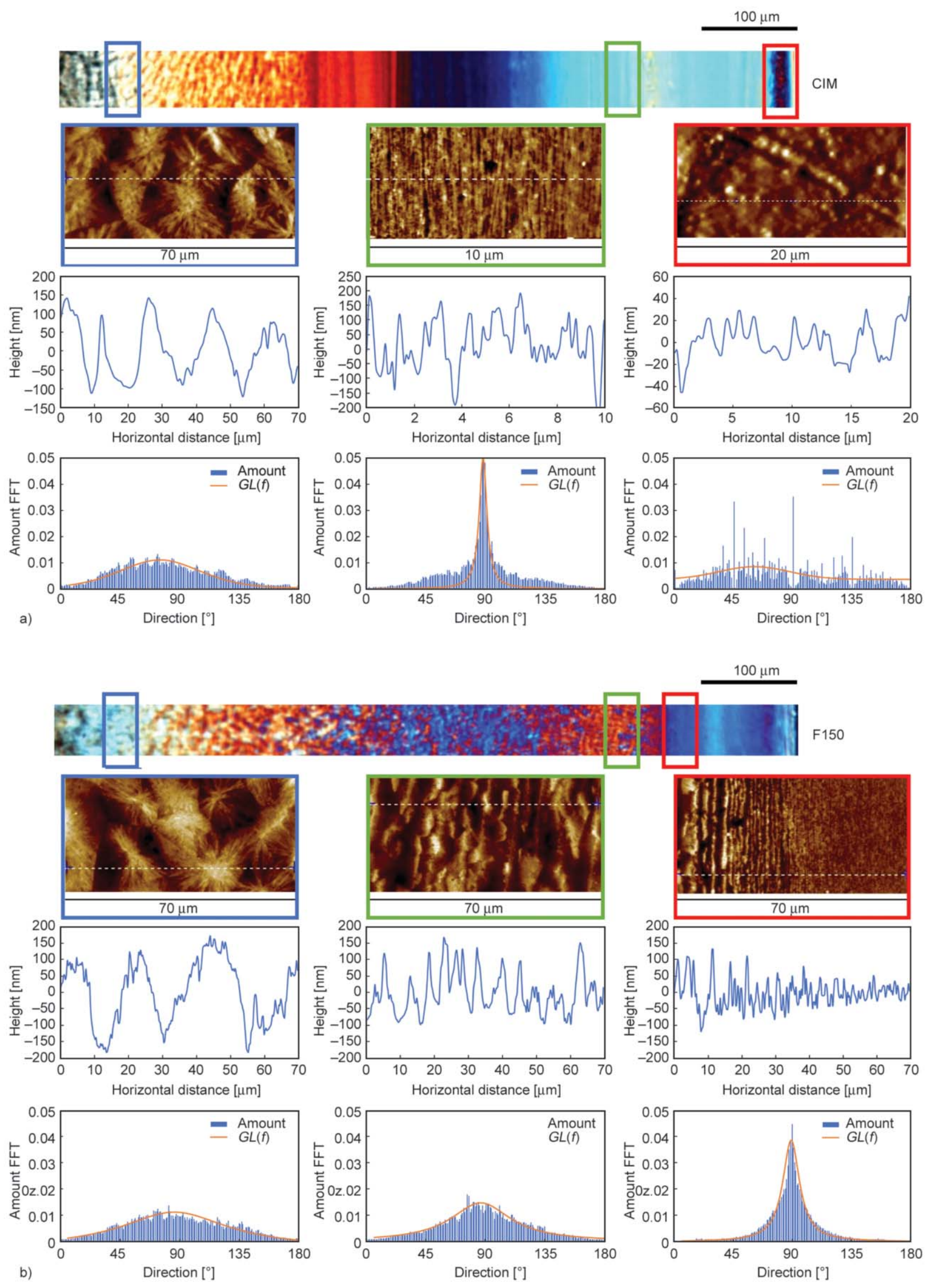

Figure 6. AFM morphology, cross-section height, and the number of structures showing alignment along a certain direction of the samples (a) CIM and (b) F150 at different distances from the sample wall. The optical micrograph is also reported for comparison. (The direction was evaluated on scan areas of $70 \mu \mathrm{m}$ wide in the spherulitical core and the transition zone, $10 \mu \mathrm{m}$ wide in the shear layer). 

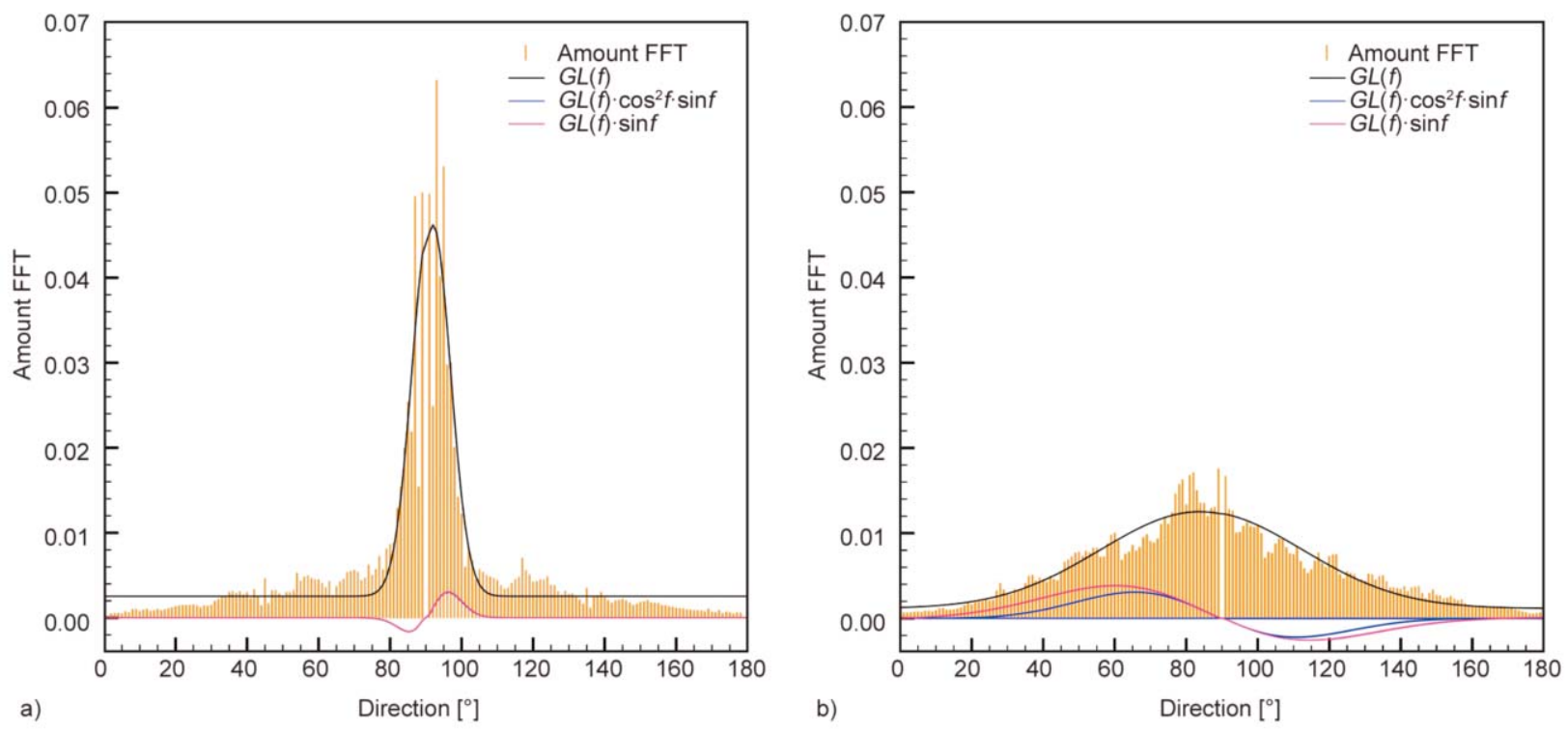

Figure 7. Number of structures showing alignment along a certain direction for F150 sample: (a) 0.08 and (b) 0.23 normalized distance from the sample wall. $G L(f)$ and the terms adopted for the Equation 5 are also reported.

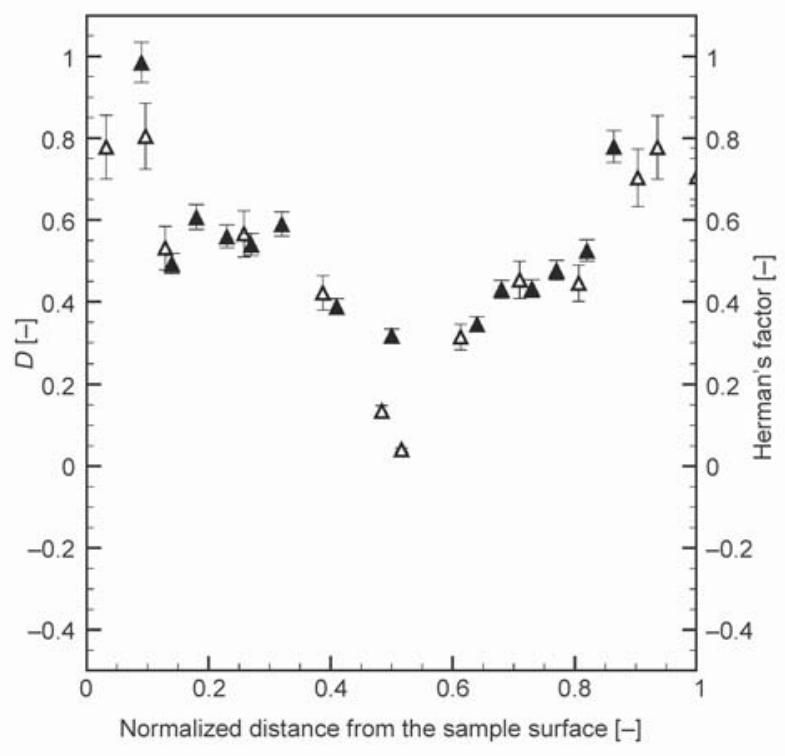

Figure 8. Comparison between the distribution along the thickness of Herman's factor evaluated from WAXS analyses and parameter $D$ for the sample F150 (full symbols are used for parameter $D$; empty symbols for Herman's factor).

comparison between Herman's factor and parameter $D$ evaluated for all the other samples.

The parameter $D$ nicely compares with Herman's factor, confirming that the developed procedure can be efficiently adopted for obtaining quantitative information about orientation from the AFM analyses. In particular, as already observed for the sample F150, the shear layer shows the highest values of $D$ (in the range $0.8-1$, the same range Herman's factor). At larger distances from the sample surface, parameter $D$ decreases down to the lower values of the sample core. In particular, the CIM sample and the sample P150 (see Figure 9a and 9d) show values of $D$ close to zero, consistently with Herman's factors. The sample P80 (see Figure 9c) shows higher values of Herman's factor in the sample core than the sample P150 (0.2 and 0, respectively). The sample F80 (see Figure 9b) shows the major discrepancy between Herman's factors and the values of parameter $D$ : Herman's factor gradually decreases from the sample walls toward the sample core, whereas the parameter $D$ sharply decreases. Nevertheless, the maximum difference between the two parameters at the same position is about 0.2 .

As mentioned above, Figure 6 shows that the direction distribution becomes wider toward the sample core; in other words, the standard deviation $(S D)$ of $G L(f)$ becomes larger. It is possible to adopt the $S D$ (which is given by the Directionality plug-in of Fiji) as a simpler method to directly relate the data coming from the Directionality plug-in of Fiji to the parameter $D$. In this work, we chose a linear function to relate $S D$ (given in radians) to $D$ (Equation (7)):

$D=1-2 b \cdot S D$

where the constant $b\left(0.4 \mathrm{rad}^{-1}\right)$ has been found to minimize the difference between Herman's factor and the parameter $D$.

As reported in Figure 10, the distributions of the parameter $D$ calculated by Equation (7) are very similar 

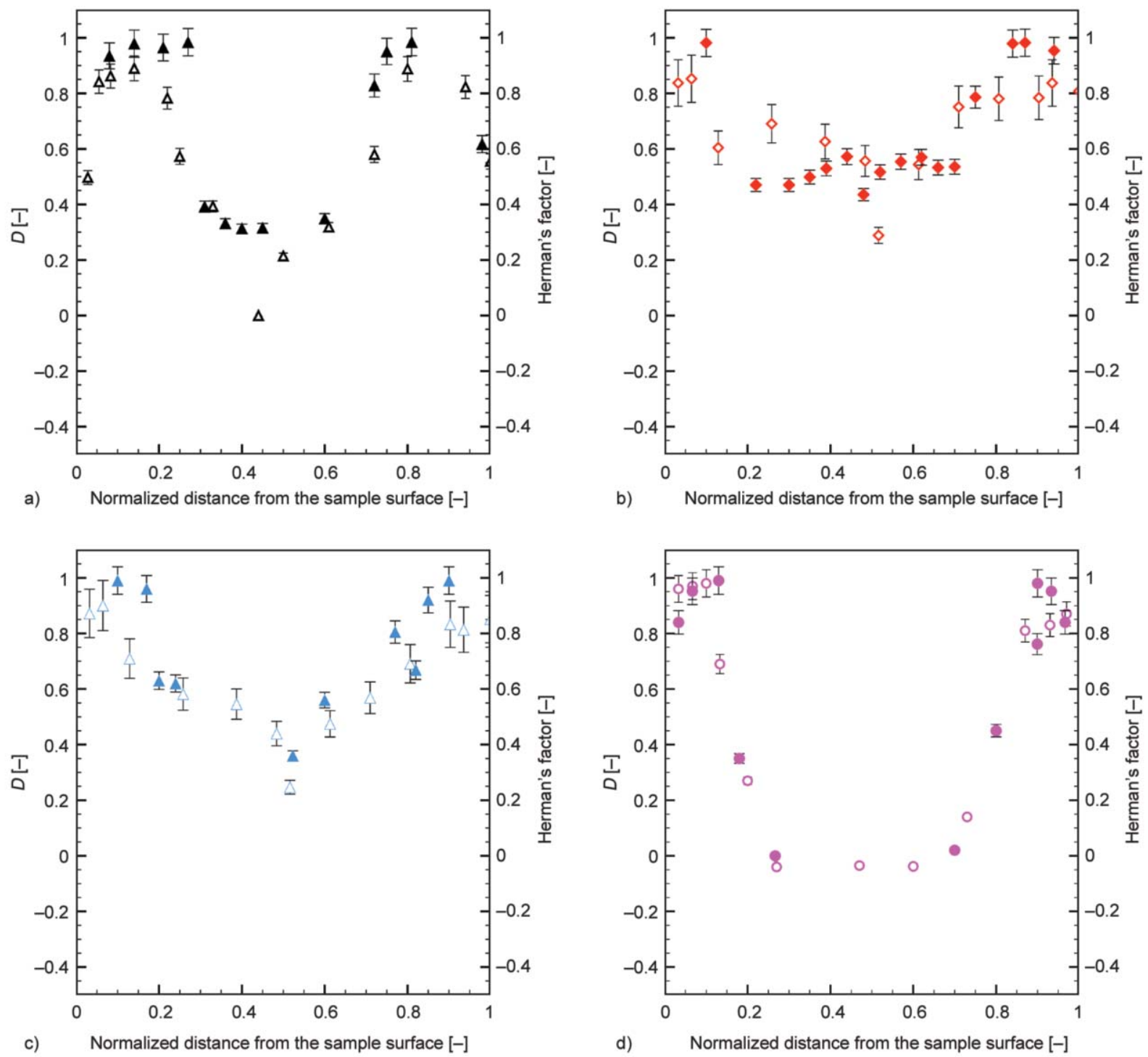

Figure 9. Comparison between the distribution along with the thickness of Herman's factor evaluated from WAXS analyses, and parameter $D$ evaluated for (a) CIM; (b) F80; (c) P80; (d) P150 (full symbols are used for the parameter D; empty symbols for Herman's factor).

to Herman's factor distributions for all the samples. This holds true also for the sample F150, which is not shown in Figure 10. The distribution of the parameter $D$ for the sample F80 (see Figure 10b) is even more consistent with Herman's factor distribution than the one observed in Figure 9: parameter $D$ almost gradually decreases from the high value at the sample surface (around 0.95) to the small values of the core.

\section{Conclusions}

The orientation of semicrystalline polymer is a key parameter in determining the final properties of the parts induced by processing conditions. To quantify the orientation within polymer parts, time-consuming techniques and complex devices are required.
A simpler method, based on atomic force microscopy, was introduced to quantify the molecular orientation and tested on molded samples obtained with different cavity surface temperature conditions. The samples were selected to present different morphologies and orientation distributions induced by the cavity temperature cycles.

The Fast Fourier Transform was applied to the AFM acquired maps and analyzed by the Directionality plug-in of Fiji. Orientation distributions were obtained for each map: where fibrils, oriented along with the flow direction, were detected, narrow distributions were found; toward the core, where more isotropic structures developed, namely spherulites, broad distributions were found. The orientations distributions were analyzed following two approaches: 

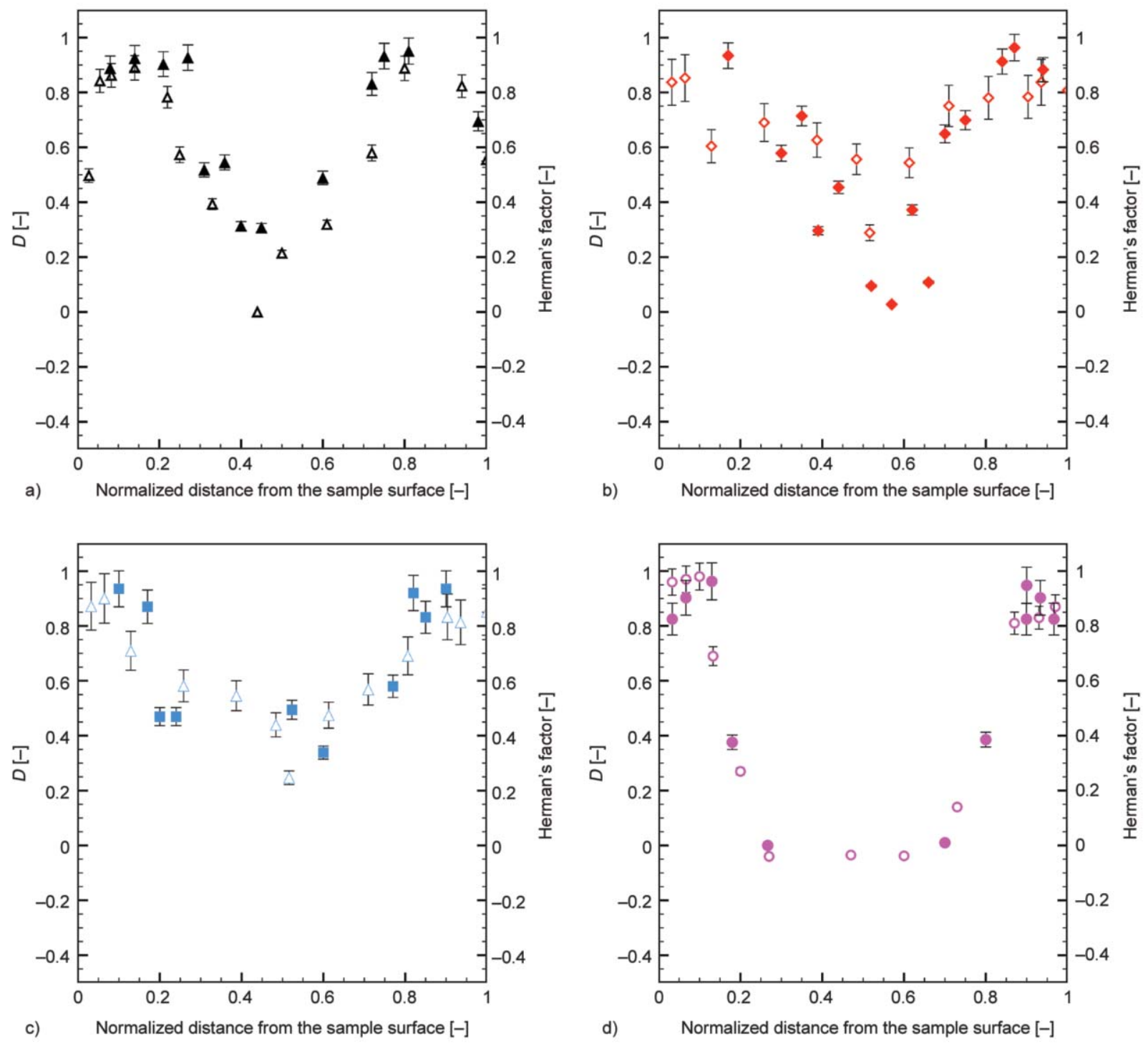

Figure 10. Comparison between the distribution along with the thickness of the Herman's factor evaluated from WAXS analyses and parameter $D$ evaluated from Equation (7) for (a) CIM; (b) F80; (c) P80; (d) P150 (full symbols are used for the parameter $D$; empty symbols for the Herman's factor).

the first one is based on Herman's analysis, the second one is based on the standard deviation of the distribution. These approaches allowed obtaining the distribution of a parameter $D$, the introduced direction parameter, along with the sample thickness. The parameter $D$ assumes zero value where a preferential direction does not characterize structures; the parameter $D$ is equal to 1 where structures develop along with a preferential direction.

The parameter $D$ was found to be sensitive to the morphology developed in the molded samples: the highest values were found where fibrils were detected, whereas the lowest ones were found for the spherulitic regions. Intermediate values of parameter
$D$ characterize the transitional regions: it gradually decreased from the high values of the fibrils to the low values of the spherulites. It was shown that the parameter $D$ is consistent with Herman's factor, evaluated by WAXS analyses, adopting both approaches and for all the considered samples.

\section{References}

[1] Hikima Y., Morikawa J., Kazarian S. G.: Analysis of molecular orientation in polymeric spherulite using polarized micro attenuated total reflection Fourier transform infrared (ATR-FTIR) spectroscopic imaging. Analytica Chimica Acta, 1065, 79-89 (2019). https://doi.org/10.1016/j.aca.2019.02.017 
[2] Rödlmeier T., Marszalek T., Held M., Beck S., Müller C., Eckstein R., Morfa A. J., Lovrincic R., Pucci A., Lemmer U., Zaumseil J., Pisula W., Hernandez-Sosa G.: Controlled molecular orientation of inkjet printed semiconducting polymer fibers by crystallization templating. Chemistry of Materials, 29, 10150-10158 (2017). https://doi.org/10.1021/acs.chemmater.7b03948

[3] Liu J., Lin D. Y., Wei B., Martin D. C.: Single electrospun PLLA and PCL polymer nanofibers: Increased molecular orientation with decreased fiber diameter. Polymer, 118, 143-149 (2017).

https://doi.org/10.1016/j.polymer.2017.04.070

[4] Tan E. P. S., Lim C. T.: Physical properties of a single polymeric nanofiber. Applied Physics Letters, 84, 16031605 (2004).

https://doi.org/10.1063/1.1651643

[5] Papkov D., Zou Y., Andalib M. N., Goponenko A., Cheng S. Z. D., Dzenis Y. A.: Simultaneously strong and tough ultrafine continuous nanofibers. ACS Nano, 7, 3324-3331 (2013). https://doi.org/10.1021/nn400028p

[6] Yue P.: Molecular orientation distribution in PC products analyzed by birefringence. Physica B: Condensed Matter, 563, 56-61 (2019).

https://doi.org/10.1016/j.physb.2019.03.033

[7] Liparoti S., Sorrentino A., Guzman G., Cakmak M., Titomanlio G.: Fast mold surface temperature evolution: Relevance of asymmetric surface heating for morphology of iPP molded samples. RSC Advances, 5, 3643436448 (2015).

https://doi.org/10.1039/C5RA04383B

[8] Xu K., Gao C., Chen G., Qiu D.: Direct evidence for effect of molecular orientation on thermoelectric performance of organic polymer materials by infrared dichroism. Organic Electronics, 31, 41-47 (2016).

https://doi.org/10.1016/j.orgel.2016.01.013

[9] Wrobel T. P., Mukherjee P., Bhargava R.: Rapid visualization of macromolecular orientation by discrete frequency mid-infrared spectroscopic imaging. Analyst, 142, 75-79 (2017).

https://doi.org/10.1039/C6AN01086E

[10] Jurczuk K., Galeski A., Mackey M., Hiltner A., Baer E.: Orientation of PVDF $\alpha$ and $\gamma$ crystals in nanolayered films. Colloid and Polymer Science, 293, 1289-1297 (2015).

https://doi.org/10.1007/s00396-015-3542-7

[11] Liparoti S., Speranza V., Titomanlio G., Pantani R.: Effect of rapid mold heating on the structure and performance of injection-molded polypropylene. Polymers, 12, 341/1-341/20 (2020).

https://doi.org/10.3390/polym12020341
[12] Pantani R., Speranza V., Titomanlio G.: Effect of flowinduced crystallization on the distribution of spherulite dimensions along cross section of injection molded parts. European Polymer Journal, 97, 220-229 (2017). https://doi.org/10.1016/j.eurpolymj.2017.10.012

[13] Speranza V., Liparoti S., Volpe V., Titomanlio G., Pantani R.: Modelling of morphology development towards spherulites and shish-kebabs: Application to isothermal flow-induced crystallization experiments on isotactic polypropylene. Polymer, 196, 122459/1-122459/12 (2020).

https://doi.org/10.1016/j.polymer.2020.122459

[14] Liparoti S., Sorrentino A., Titomanlio G.: Fast cavity surface temperature evolution in injection molding: Control of cooling stage and final morphology analysis. RSC Advances, 6, 99274-99281 (2016).

https://doi.org/10.1039/C6RA22968A

[15] Speranza V., Liparoti S., Pantani R., Titomanlio G.: Hierarchical structure of iPP during injection molding process with fast mold temperature evolution. Materials, 12, 424/1-424/16 (2019).

https://doi.org/10.3390/ma12030424

[16] Wilchinsky Z. W.: Orientation in cold-rolled polypropylene. Journal of Applied Polymer Science, 7, 923-933 (1963).

https://doi.org/10.1002/app.1963.070070311

[17] García R., Magerle R., Perez R.: Nanoscale compositional mapping with gentle forces. Nature Materials, 6 , 405-411 (2007).

https://doi.org/10.1038/nmat1925

[18] García R., Pérez R.: Dynamic atomic force microscopy methods. Surface Science Reports, 47, 197-301 (2002). https://doi.org/10.1016/S0167-5729(02)00077-8

[19] Horcas I., Fernández R., Gómez-Rodríguez J. M., Colchero J., Gómez-Herrero J., Baro A. M.: WSXM: A software for scanning probe microscopy and a tool for nanotechnology. Review of Scientific Instruments, 78, 013705/1-013705/8 (2007). https://doi.org/10.1063/1.2432410

[20] Liu Z-Q.: Scale space approach to directional analysis of images. Applied Optics, 30, 1369-1373 (1991). https://doi.org/10.1364/AO.30.001369

[21] Somani R. H., Yang L., Zhu L., Hsiao B. S.: Flow-induced shish-kebab precursor structures in entangled polymer melts. Polymer, 46, 8587-8623 (2005). https://doi.org/10.1016/j.polymer.2005.06.034

[22] Phulkerd P., Hirayama S., Nobukawa S., Inoue T., Yamaguchi M.: Structure and mechanical anisotropy of injection-molded polypropylene with a plywood structure. Polymer Journal, 46, 226-233 (2014). https://doi.org/10.1038/pj.2013.88 